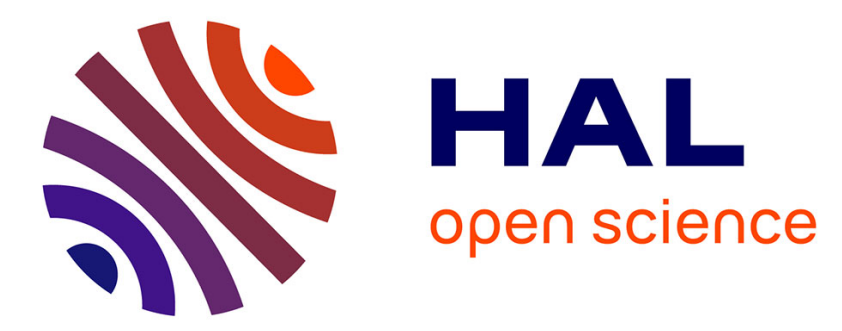

\title{
LE DÉVELOPPEMENT DE L'ENSEIGNEMENT SECONDAIRE EN TANZANIE ET LA SCOLARISATION DES MAASAI
}

\author{
Nathalie Bonini
}

\section{- To cite this version:}

Nathalie Bonini. LE DÉVELOPPEMENT DE L'ENSEIGNEMENT SECONDAIRE EN TANZANIE ET LA SCOLARISATION DES MAASAI. Autrepart - Revue de sciences sociales au Sud, 2011, 3 (59), pp.57-74. 10.3917/autr.059.0057 . hal-01090275

\section{HAL Id: hal-01090275 https://hal.science/hal-01090275}

Submitted on 4 Dec 2014

HAL is a multi-disciplinary open access archive for the deposit and dissemination of scientific research documents, whether they are published or not. The documents may come from teaching and research institutions in France or abroad, or from public or private research centers.
L'archive ouverte pluridisciplinaire HAL, est destinée au dépôt et à la diffusion de documents scientifiques de niveau recherche, publiés ou non, émanant des établissements d'enseignement et de recherche français ou étrangers, des laboratoires publics ou privés. 


\title{
LE DÉVELOPPEMENT DE L'ENSEIGNEMENT SECONDAIRE EN TANZANIE ET LA SCOLARISATION DES MAASAI
}

\author{
Nathalie Bonini \\ Presses de Sciences Po | Autrepart
}

\author{
$2011 / 3-N^{\circ} 59$ \\ pages 57 à 74
}

ISSN 1278-3986

Article disponible en ligne à l'adresse:

http://www.cairn.info/revue-autrepart-2011-3-page-57.htm

Pour citer cet article :

Bonini Nathalie , «Le développement de l'enseignement secondaire en Tanzanie et la scolarisation des Maasai », Autrepart, 2011/3 №59, p. 57-74. DOI : 10.3917/autr.059.0057

Distribution électronique Cairn.info pour Presses de Sciences Po.

(C) Presses de Sciences Po. Tous droits réservés pour tous pays.

La reproduction ou représentation de cet article, notamment par photocopie, n'est autorisée que dans les limites des conditions générales d'utilisation du site ou, le cas échéant, des conditions générales de la licence souscrite par votre établissement. Toute autre reproduction ou représentation, en tout ou partie, sous quelque forme et de quelque manière que ce soit, est interdite sauf accord préalable et écrit de l'éditeur, en dehors des cas prévus par la législation en vigueur en France. II est précisé que son stockage dans une base de données est également interdit. 


\title{
Le développement de l'enseignement secondaire en Tanzanie et la scolarisation des Maasai
}

\author{
Nathalie Bonini*
}

Pour atteindre les objectifs fixés lors du forum de l'Éducation pour tous (EPT) de Dakar (2000) et ceux du Millénaire pour le développement (OMD) à l'horizon 2015, le gouvernement tanzanien a adopté une série de réformes qui ont à la fois accru le taux de scolarisation et modifié le paysage éducatif. Si le développement du premier degré demeure une priorité nationale, celui-ci n'est plus hégémonique ; en témoigne le récent accroissement, sans précédent depuis l'indépendance, du taux de scolarisation au niveau secondaire, même s'il demeure toujours parmi les plus bas du continent africain. Pratiquement inaccessible pour les Africains sous la colonisation, l'éducation secondaire n'a jamais constitué une priorité du gouvernement tanzanien qui, en dehors d'une courte période post-indépendance, a largement concentré ses efforts sur le cycle primaire. En effet, c'est sur l'éducation du plus grand nombre en relation étroite avec l'agriculture, pilier du développement économique du pays, que s'est édifiée la Tanzanie socialiste indépendante. Selon la philosophie de l' « éducation pour l'autosuffisance » (education for self reliance) formulée lors de la déclaration d'Arusha en 1967, et qui a durablement et profondément marqué le système éducatif ${ }^{1}$, l'école devait servir la collectivité avant d'être envisagée comme un moyen de promotion individuelle. Au-delà du primaire, le système devenait très sélectif, laissant à chaque étape du cursus un nombre important de jeunes gens non diplômés et une part non négligeable d'élèves qui, malgré leur réussite à l'examen, n'intégraient pas le niveau supérieur. La primauté accordée à l'éducation de base a porté ses fruits, la scolarisation primaire universelle ayant pratiquement été atteinte à l'aube des années 1980, mais elle a contribué à creuser un large fossé entre les premier et second degrés ${ }^{2}$. La profonde crise économique qu'a connue la Tanzanie dans les années 1980 et les plans d'ajustement structurel qui ont suivi ont eu des répercussions importantes, notamment sur le cycle primaire qui a davantage pâti de la crise et des coupes budgétaires; ces dernières ont moins affecté le cycle secondaire qui recevait

* Université de Tours, UMR 6173, CITERES.

1. Pour une analyse plus détaillée du système éducatif tanzanien, $c f$. Bonini [2003].

2. Il n'en fut pas de même au Kenya voisin où la volonté similaire de développer surtout l'éducation de base n'a pas atrophié la scolarisation secondaire [Charton, 2003]. 
une maigre part des sommes allouées à l'éducation. Conjuguée à la forte demande sociale d'éducation post-primaire, la volonté de réduire les dépenses d'éducation a incité le gouvernement à libéraliser le développement du secteur secondaire privé dont il avait longtemps freiné l'expansion, considérant qu'elle serait source d'inégalités, car davantage susceptible d'advenir dans les régions scolairement et économiquement privilégiées. Ainsi, alors que le taux de scolarisation dans l'enseignement primaire a sévèrement décliné des années 1980 à la fin du siècle, celui du secondaire s'est légèrement accru. Cette progression s'est amplifiée suite aux divers plans d'éducation mis en œuvre au milieu des années 2000, qui ont conduit à l'amélioration de l'infrastructure scolaire sur tout le territoire.

Comme leurs compatriotes, mais dans une proportion nettement moindre, les pasteurs maasai ont profité du développement de la scolarisation au-delà du primaire. Conformément à la politique de décentralisation, les nouvelles écoles secondaires gouvernementales construites dans la région sont essentiellement des écoles communautaires, principalement financées par les familles. Les enfants maasai qui, lorsqu'ils intégraient le circuit scolaire, ne poursuivaient pratiquement jamais audelà de l'école primaire, peuvent désormais le faire. Toutefois, non seulement ils demeurent parmi les moins scolarisés du pays, mais leurs conditions d'apprentissage figurent aussi au rang des plus mauvaises. Par ailleurs, si, comme nous le verrons la paupérisation de nombre d'entre eux, liée essentiellement au déclin du pastoralisme, les incite à scolariser leurs enfants pour diversifier leurs ressources, elle se révèle une contrainte forte pour les amener ou les maintenir à l'école secondaire.

Cet article, qui se fonde sur des enquêtes de terrain réalisées entre 1991 et 1993 puis de nouveau en 2009, se propose d'étudier l'évolution de la scolarisation des Maasai au regard de celle qui prévaut dans l'ensemble du pays depuis la fin des années 1990. La première partie sera consacrée aux politiques éducatives nationales et à l'accroissement du taux de scolarisation qui en découle, tandis que la deuxième partie mettra en évidence les inégalités sociales et régionales de l'accès à l'éducation. Réduisant ensuite la focale, la troisième partie se centrera sur les effets de ces réformes en matière d'offre scolaire et de conditions de scolarisation dans les zones rurales du nord du pays peuplées par les Maasai. Confrontant cette offre scolaire à la demande d'éducation, nous verrons que si la possibilité accrue de poursuivre une scolarité au-delà du primaire modifie les attentes à l'égard de l'école comme les pratiques éducatives des Maasai, celles-ci sont fortement dépendantes de leur situation économique.

\section{Les réformes éducatives en Tanzanie}

\section{L'organisation du système éducatif}

Obligatoire à partir de 7 ans, l'enseignement primaire se compose de sept niveaux, désignés par le terme anglais standard ${ }^{3}$. Il se clôt par un examen national,

3. La première classe de l'enseignement primaire est le standard 1 et la dernière, le standard 7. Depuis le milieu des années 2000, la plupart des écoles - idéalement toutes - ont ouvert un niveau inférieur de «préscolarisation » pour accueillir les enfants dès 6 ans. 
le Primary School Leaving Examination (PSLE) qui, dernier d'une série d'examens à l'échelle locale et régionale, détermine la possibilité de poursuivre la scolarité dans les écoles secondaires publiques. En effet, seules les écoles privées accueillent les élèves non diplômés. Il est théoriquement impossible de redoubler la dernière année du primaire et de retenter l'examen, même s'il arrive fréquemment que cette interdiction soit contournée ${ }^{4}$. Le comité national de sélection (National Examination Council) examine les dossiers des élèves reçus et, en fonction de leurs notes, les affecte dans les différentes écoles gouvernementales, les plus prestigieuses étant en principe réservées aux meilleurs élèves. Les écoles secondaires publiques sont en effet de deux types: les écoles à rayonnement national ou régional, et les écoles communautaires, construites par les habitants mais gérées par le gouvernement. Moins prestigieuses et pour beaucoup, plus récentes, ces dernières ont un recrutement essentiellement local. Les écoles privées sont plus variées mais la plupart - les plus nombreuses - sont confessionnelles ou gérées par des ONG.

L'enseignement secondaire est divisé en deux cycles : le secondaire de base (lower secondary) d'une durée de quatre ans (de form 1 à form 4) et le secondaire supérieur (upper secondary) de deux ans (forms 5 et 6). Les écoles offrant les six années de second cycle ne représentaient à la fin des années 1990 que $10 \%$ des écoles secondaires [Lassibille, Tan, Sumra, 2000, p. 8]. Du swahili dans le cycle primaire, la langue d'instruction devient l'anglais - qui n'était alors enseigné que comme langue étrangère - dès la première année du secondaire ; il en est de même pour les manuels scolaires. Fondé sur le modèle du O-level britannique, le curriculum est à la fois national et spécialisé dans la mesure où chaque école ne propose qu'une partie des options du programme ${ }^{5}$. À la fin de la deuxième année, les élèves passent un examen qui les autorise à s'inscrire dans l'année supérieure. Ils peuvent néanmoins redoubler en cas d'échec et tenter de nouveau l'examen. Le passage dans le cycle secondaire supérieur est conditionné à la réussite à l'examen national, le Certificate of Secondary Education Examination (CSEE) qui se déroule à la fin de la $4^{\mathrm{e}}$ année. Pour réussir, les élèves doivent obtenir des notes entre $\mathrm{A}$ et $\mathrm{C}$ dans au moins cinq des neuf disciplines évaluées ${ }^{6}$. Le secondaire supérieur se termine lui aussi par un examen, l'Advanced Certificate of Secondary Education (ACSE), qui permet de poursuivre des études à l'université ou dans d'autres institutions supérieures. En revanche, il n'est pas nécessaire d'avoir accompli les six années de scolarisation secondaire, et a fortiori d'en être diplômé, pour accéder aux écoles de formation des enseignants. Les 34 établissements publics de formation des maîtres (Teacher Colleges) présents en 2000 sont en effet ouverts à tous les élèves sortant de la $4^{\mathrm{e}}$ année de l'enseignement secondaire ainsi qu'aux

4. En cas d'échec à l'examen, les parents peuvent par exemple inscrire leur enfant sous un autre nom dans une autre école, soit en dernière année de cycle, soit le plus souvent dans le niveau qui précède pour éviter que l'élève soit reconnu, l'administration disposant des photos de chaque élève du standard 7.

5. Les écoles privées suivent globalement le même programme et sont, comme les établissements publics, inspectées par des inspecteurs nommés par le gouvernement.

6. Le système de notation va de $\mathrm{A}$ (la meilleure note) à $\mathrm{E}$ (la plus mauvaise). 
enseignants déjà en poste mais non formés [MEC, 2001, p. 1]. Le programme de formation continue des maîtres «In-Service programme » a été mis en place à la fin des années 1990 pour améliorer la formation des nombreux enseignants du primaire qui ne disposent pour tout bagage scolaire que du certificat de fin d'études primaires (PSLE). Les études durent deux ans et incluent un enseignement académique et professionnel; à l'issue de ces deux années, et en fonction de leur niveau scolaire de départ, les élèves obtiennent un certificat de grade $\mathrm{A}$ (grade A certificate) ou le grade supérieur, le diplôme (Diploma), les premiers étant affectés dans les écoles primaires tandis que les seconds pourront intégrer les écoles secondaires.

\section{L'évolution de l'offre scolaire depuis les années 2000}

Souhaitant enrayer la chute du taux de scolarisation primaire et encouragé par les bailleurs de fonds, le gouvernement tanzanien a lancé une série de réformes au début des années 2000. La première, le Primary Education Development Plan (PEDP) 2002-2006 concerne le cycle primaire avec pour mesure phare, l'abolition, pour la rentrée scolaire 2001-2002, des frais de scolarité qui avaient été rétablis en 1994 après vingt ans de gratuité. Cette mesure a été suivie d'un afflux massif d'élèves à ce niveau. Le taux net de scolarisation primaire qui n'avait cessé de chuter depuis les années 1980 a ainsi fortement augmenté cette dernière décennie (tableau 1).

Tableau 1 - Taux de scolarisation dans l'enseignement primaire en Tanzanie

\begin{tabular}{l|c|c|c|c}
\hline Années & $\mathbf{1 9 8 1}$ & $\mathbf{2 0 0 0}$ & $\mathbf{2 0 0 6}$ & $\mathbf{2 0 1 0}$ \\
\hline Taux nets de scolarisation & $68 \%$ & $59 \%$ & $96 \%$ & $95 \%$ \\
\hline Taux bruts de scolarisation & $94 \%$ & $78 \%$ & $118 \%$ & $106 \%$ \\
\hline
\end{tabular}

Sources : MoEVT, [2010] ; Unesco, [2000] ; Woods, [2007].

Après avoir été le parent pauvre de l'éducation pendant des décennies ${ }^{7}$, le cycle secondaire est encadré en 2004 par le Secondary Education Development Plan (SEDP). Comme indiqué en préambule de ce plan, l'expansion de l'école secondaire est désormais jugée nécessaire pour accompagner la progression attendue du taux de scolarisation primaire - à la fois en terme de débouchés scolaires pour les élèves et en terme d'encadrement - de façon à répondre à la demande croissante d'éducation post-primaire de la population, à mieux satisfaire les besoins en main-d'œuvre qualifiée de l'économie moderne, et à réduire la pauvreté [MEC, 2004]. Les justifications d'ordre économique qui lient le développement de l'enseignement secondaire aux emplois salariés et au besoin de

7. Les inscriptions dans les écoles gouvernementales primaires ont quintuplé entre 1965 et 1981, alors qu'elles n'ont augmenté que de $81 \%$ dans les secondaires gouvernementales [Samoff, 1987, p. 340]. 
développer le secteur tertiaire et l'enseignement supérieur constituent une rupture radicale avec la philosophie de l'éducation pour l'autosuffisance, qui considérait que l'éducation devait favoriser le développement rural et constituer une école de la vie plutôt qu'une préparation aux études supérieures [Bonini, 2003 ; Wedgwood, 2005].

Comme nous l'avons noté précédemment, à la fin des années 1980, le gouvernement avait stimulé l'implantation d'écoles secondaires privées, qui scolarisaient environ la moitié des élèves de ce niveau à la fin du siècle. L'expansion du secteur privé a considérablement diminué au profit du secteur public après la mise en place du SEDP en 2004 et la construction de plus d'un millier d'écoles secondaires gouvernementales - pour la plupart communautaires - entre 2003 et 2006. Cette progression s'est poursuivie les années suivantes, le nombre d'écoles secondaires gouvernementales passant de 829 en 2004 à 3397 en 2010 (au cours de la même période, le nombre d'écoles secondaires non gouvernementales est passé de 462 à 869) [MoEVT, 2010, p. 67].

Les principales sources de financement de ce plan proviennent en premier lieu du budget du gouvernement - qui continue de réserver la plus grosse part à l'éducation de base ${ }^{8}-$ des prêts et dons de différents partenaires du développement, des contributions des habitants [MEC, 2004] ${ }^{9}$ et, dans une moindre mesure et aléatoirement, de l'autofinancement provenant le plus souvent des revenus tirés des champs des écoles.

Les effectifs du secondaire ont pu augmenter grâce à la progression des taux de réussite à l'examen de fin de primaire (tableau 2) et à la progression du taux de transition du primaire au secondaire.

Tableau 2 - Taux de réussite à l'examen PSLE de fin de cycle primaire

\begin{tabular}{l|c|c|c|c|c|c|c}
\hline Années & $\mathbf{1 9 9 8}$ & $\mathbf{2 0 0 1}$ & $\mathbf{2 0 0 3}$ & $\mathbf{2 0 0 6}$ & $\mathbf{2 0 0 7}$ & $\mathbf{2 0 0 8}$ & $\mathbf{2 0 0 9}$ \\
\hline Taux de réussite & $21,3 \%$ & $28,6 \%$ & $40,0 \%$ & $70,5 \%$ & $54,1 \%$ & $53,7 \%$ & $49,4 \%$ \\
\hline
\end{tabular}

Sources : MEC [2004]; MoEVT [2010b, p. 9].

La forte croissance du taux de réussite à partir de 2003 tient en partie aux modifications du contrôle des connaissances cette année-là : alors que les élèves devaient auparavant composer dans trois matières (langue, science et mathématiques), le swahili a été ajouté à liste des enseignements évalués, ce qui a eu pour effet de réduire le poids des mathématiques, où les élèves avaient tendance à

8. Pour information, il était en 2000 de $66 \%$ contre $20 \%$ pour l'éducation supérieure et seulement $7,2 \%$ pour le cycle secondaire. [MEC, 2000, p. 15]. Par ailleurs, plus de la moitié du budget gouvernemental de l'éducation secondaire est dédiée aux dépenses de personnels et plus du quart à celles de la restauration.

9. Selon un rapport du ministère de l'Education nationale, seuls $75 \%$ des frais de scolarité sont effectivement perçus [MoEVT, 2010b, p. 10]. 
échouer [Davidson 2005 cité par Wedgwood, 2005, p. 30] ${ }^{10}$. Le taux de transition du primaire au secondaire, qui était de $20 \%$ en 1967, puis était descendu à $7 \%$ en 1980 [Al-Samarrai, Reilly, 2005, p. 3], est passé à 15,4\% en 1990, 19,1\% en 1998, 21,7\% en 2003, et autour de $25 \%$ en 2006 [MEC, 2000 ; MEC, 2004 ; Woods, 2007]. Ce taux de transition dépend du nombre d'élèves ayant réussi l'examen national de fin de primaire, de la demande d'éducation post-primaire et des places disponibles dans les écoles secondaires.

Demeuré aux alentours de $3 \%$ de 1970 au milieu des années 1980, le taux net de scolarisation dans le cycle secondaire a progressé pendant les deux décennies suivantes (pour atteindre $4 \%$ en 1988 et $6 \%$ en 2000) puis, comme le montre le tableau 3, il a ensuite doublé en six ans et accéléré sa progression au cours des trois années suivantes, atteignant 27,8 \% en 2009 [MoEVT, 2010b, p. 7 ; Woods, 2007, p. 5].

Tableau 3 : Taux de scolarisation dans l'enseignement secondaire

\begin{tabular}{l|l|c|c|c|c}
\hline Cycles & $\begin{array}{c}\text { Taux } \\
\text { de scolarisation }\end{array}$ & $\mathbf{2 0 0 0}$ & $\mathbf{2 0 0 4}$ & $\mathbf{2 0 0 6}$ & $\mathbf{2 0 0 9}$ \\
\hline $\begin{array}{l}\text { Secondaire de base } \\
\text { (classes 1- 4) }\end{array}$ & $\begin{array}{l}\text { Taux net } \\
\text { de scolarisation } \\
\text { Taux brut } \\
\text { de scolarisation }\end{array}$ & - & $8,40 \%$ & - & $29,10 \%$ \\
\hline $\begin{array}{l}\text { Ensemble du secondaire } \\
\text { (classes 1- 6) }\end{array}$ & $\begin{array}{l}\text { Taux net } \\
\text { de scolarisation } \\
\text { Taux brut } \\
\text { de scolarisation }\end{array}$ & $6,00 \%$ & $5,90 \%$ & $13,10 \%$ & $27,80 \%$ \\
\hline
\end{tabular}

Sources : MoEVT [2010b, p. 7]; Woods [2007, p. 5].

Les déperditions dans le premier cycle du secondaire demeurent élevées : des études réalisées sur la cohorte d'élèves entrés en première année de cycle secondaire en 2000 révèlent que moins de $72 \%$ d'entre eux sont présents en dernière année, quatre ans plus tard [MEC, 2004]. Par ailleurs, malgré la progression du taux de réussite à l'examen CSEE (39\% en 2004 contre 21,3\% en 1999) [Wedgwood, 2005, p. 33], tous les lauréats ne sont pas assurés de poursuivre leur scolarité dans le second cycle du secondaire public et une fois ce niveau atteint, le taux d'abandon au cours des deux années de ce cycle est très supérieur à celui du secondaire de base. Si le plan SEDP de 2004 insiste sur la nécessité de fournir

10. Pour Sumra et Rajani, le mode d'évaluation de l'examen PLSE ne permet pas réellement d'évaluer la capacité des élèves à poursuivre leurs études : «Les examens se déroulent sous la forme de questions à choix multiples qui, pour une large part, mesurent la mémorisation de faits. Même les tests d'anglais et de swahili ne requièrent pas des élèves qu'ils écrivent la moindre phrase ! » [2006, p. 5]. Traduction de l'auteur. Les autres traductions de citations d'auteurs anglophones dans le texte sont également de l'auteur. 
un enseignement de qualité, les pressions politiques en faveur de la quantité sont importantes, notamment en raison de l'arrivée prévue en 2008 de la cohorte des élèves entrés en primaire au moment de l'abolition des droits d'inscription en 2001 et de l'objectif annoncé pour l'horizon 2010 d'un taux de transition de $50 \%$ entre le primaire et le secondaire d'une part, et entre le secondaire de base et le secondaire supérieur d'autre part [Wedgwood, 2005, p. 41]. Comme le soulignent différents auteurs [Wedgwood, 2005 ; Sumra, Rajani, 2006 ; MoEVT, 2010b], la rapide expansion de l'enseignement secondaire a ses revers en terme de qualité, le principal étant la pénurie de professeurs, et notamment de professeurs formés et compétents pour enseigner à ce niveau. Selon les auteurs d'un rapport annuel sur l'éducation en Tanzanie : «Sur le front du secondaire, tandis qu'entre 2005 et 2009 le nombre d'écoles et d'élèves a augmenté respectivement de $135 \%$ et de $180 \%$, le nombre d'enseignants n'a crû que de $81 \%$, ce qui a pratiquement doublé le ratio enseignant-élèves dans l'école secondaire, passant de $1 / 22$ à 1/43. » [HakiElimu, 2009, p. IV] ${ }^{11}$. De plus, cette forte croissance engendre autant qu'elle masque les carences matérielles de locaux, fournitures scolaires, etc. qui sont, comme nous le montrerons ultérieurement, particulièrement criantes en milieu rural. Enfin, si l'accès au secondaire s'est ouvert, il ne s'est pas complètement démocratisé dans la mesure où les frais engendrés par la scolarisation des enfants à ce niveau sont importants, voire totalement prohibitifs.

\section{Des inégalités sociales et régionales d'accès à l'éducation}

\section{Les contraintes économiques : un frein à la scolarisation secondaire}

La fréquentation de l'école secondaire a pendant très longtemps été réservée à une élite, le plus souvent urbaine dans la mesure où les villes abritaient la majorité des établissements. Ensuite, l'augmentation du taux de scolarisation à ce niveau devant, à ses débuts, beaucoup au développement du secteur privé, n'a guère profité aux élèves issus des groupes sociaux les moins favorisés. Selon Lassibille, Tan, et Sumra, le développement du secteur privé secondaire dans les années 1980 a augmenté les inégalités sociales face à l'école : « Notre étude montre que l'expansion s'est accompagnée d'un déclin de l'accès à la scolarisation secondaire des enfants issus de milieux défavorisés et, sur le plan des écoles, par une forte instabilité du personnel.»[2000, p. 2]. Mais cette surreprésentation des élèves issus de familles aisées ne concerne pas uniquement les écoles privées qui sont les plus onéreuses. Se fondant sur des données du milieu des années 1990, Wedgwood indique que $36 \%$ des élèves des écoles secondaires gouvernementales proviennent des $20 \%$ des familles les plus riches, contre environ $8 \%$ pour le quintile le plus pauvre [Wedgwood, 2005, p. 13] ; de même, $11,3 \%$ des personnes appartenant au quintile supérieur ont un niveau de fin de secondaire contre 1,3 des $20 \%$ les plus pauvres [Wedgwood, 2005, p. 21].

11. HakiElimu est une ONG tanzanienne fondée en 2001 pour la promotion de l'éducation. 
Les frais de scolarité, qui s'élevaient jusqu'en 2004 à 70000 TZS pour les internats et $40000 \mathrm{TZS}$ pour les externats [Wedgwood, 2005, p. 13] furent réduits à 20000 TZS (environ 15 USD) en janvier 2005 [MoEVT, 2010b, p. 9]. En dehors des coûts d'opportunité, le coût de scolarisation d'un enfant à l'école secondaire était estimé en 2000 à plus de 200000 TZS, somme incluant les frais de scolarité, l'uniforme, les transports, les frais d'examens, le matériel pédagogique, l'argent de poche - notamment pour les enfants internes qui doivent payer eux-mêmes de nombreux frais annexes - voire les cours particuliers et autres dépenses [MEC, 2000, p. 16]. Ces coûts de scolarisation sont prohibitifs pour nombre de familles tanzaniennes et, s'ils ne le sont pas totalement, les empêchent de scolariser tous leurs enfants à ce niveau. À ces dépenses par enfant, il convient d'ajouter celles auxquelles les familles doivent collectivement consentir pour construire et entretenir les bâtiments scolaires, équiper les internats en matelas, participer à la rémunération des cuisiniers ou des gardiens des écoles, etc. En effet, depuis les années 1990, à l'instar des autres pays africains, et sur les recommandations de l'Unesco et des bailleurs de fonds au nom d'un partage des responsabilités et d'un accroissement de l'efficacité du système, le gouvernement tanzanien a élargi le nombre de partenaires entrant dans le financement de l'éducation, l'objectif affiché étant d'impliquer davantage les communautés. Ce principe se traduit surtout par un transfert de certaines responsabilités financières de l'État aux acteurs privés, en premier lieu les communautés et les familles.

Selon le rapport sur la démographie et la santé en Tanzanie [NBS, ORC Macro, 2004] le taux net de scolarisation (TNS) ${ }^{12}$ à l'école primaire était en 2004-2005, de $88 \%$ pour les enfants des familles les plus riches contre $58 \%$ pour les enfants des familles les plus pauvres. Cet écart s'amplifie au niveau secondaire, puisque le taux net de fréquentation était de $23 \%$ pour les enfants des familles les plus riches, de $7 \%$ pour le quatrième quintile, de $2 \%$ pour le troisième, de $1 \%$ pour le second et seulement de $0,4 \%$ pour les enfants des ménages les plus pauvres [op. cit. p. 16]. Comme le mentionnent à dix ans d'intervalle les deux plans de développement de l'enseignement secondaire [MEC, 2000 p. 21 ; MoEVT, 2010b, p. 15], pour prévenir l'accroissement des inégalités, des mesures visant à faire évoluer la composition socioéconomique des élèves doivent accompagner celles tournées vers l'amélioration des taux de scolarisation dans l'enseignement secondaire ; la création d'écoles secondaires communautaires, ou plutôt l'encouragement à leur construction, en est une. Il reste que si l'amplification du nombre d'écoles communautaires dans la deuxième moitié des années 2000 a permis d'amener sur les bancs de l'école des enfants qui en étaient auparavant exclus, du fait de leur qualité généralement moindre, elle n'a pas pour autant réduit les inégalités scolaires ni modifié leur géographie.

12. Le taux net de scolarisation est le pourcentage d'enfants d'âge scolaire (soit entre 7 et 13 ans pour le primaire, et entre 14 et 19 ans pour le secondaire) qui fréquentent l'école. Le TBS (taux de brut de scolarisation) est le pourcentage d'élèves qui fréquentent l'école, indépendamment de leur âge. Est considérée comme « fréquentant l'école » toute personne présente à l'école à n'importe quel moment de l'année [Tanzania DHS, 2004, p. 16]. 


\section{Les zones rurales défavorisées}

Sur le plan géographique, on note d'importantes inégalités régionales en matière d'infrastructures scolaires et de taux de scolarisation. À titre d'exemple, la région du Kilimanjaro, dont la supériorité en matière d'éducation scolaire remonte aux toutes premières heures de la colonisation via l'implantation de nombreuses écoles missionnaires, possédait en 2003 plus du double des écoles de n'importe quelle autre région et trois fois plus que la plupart d'entre elles [Wedgwood, 2005, p. 13]. S' agissant des écoles secondaires, cette suprématie numérique de la région dans les secteurs public et privé - toutefois atténuée depuis la création à la fin des années 2000 d'écoles communautaires dans les cantons qui en étaient dépourvus - se double d'une meilleure réputation des établissements, qui sont mieux pourvus en personnels qualifiés et plus nombreux à proposer un enseignement incluant les six années du secondaire. L'écart entre les régions se mesure également en termes du taux de fréquentation scolaire : le TNS pour l'école secondaire s'élevait en 2004-2005 à près de $20 \%$ à Dar es Salaam, $17 \%$ dans la région du Kilimanjaro, contre 0,3\% dans la région de Tabora [NBS, ORC Macro, 2004, p. 18].

Pour pallier les inégalités de réussite à l'examen de fin de primaire entre les régions scolairement favorisées et les autres, un système de quotas d'accès aux écoles secondaires gouvernementales par région a été instauré à la fin des années 1990. Un nombre de places déterminé dans les écoles secondaires du secteur public a été alloué à chacun des 113 districts : concrètement, les élèves des régions ou districts favorisés devaient, pour avoir une place dans une école secondaire publique, obtenir une meilleure note que ceux qui avaient passé l'examen dans un district défavorisé [Lassibille, Tan, Sumra, 2000 ; Wedgwood, 2005]. Cette mesure a été en partie détournée, certaines familles envoyant leurs enfants en dernière année du primaire dans les écoles des zones dont les quotas étaient les plus élevés, de façon à optimiser leurs chances d'accéder à une école secondaire publique. Aujourd'hui, le système de quotas régionaux perdure pour l'accès, sélectif, aux écoles gouvernementales régionales, pourvues d'internats, mais la plupart des places à l'école secondaire sont attribuées à l'intérieur du district, ce qui limite l'impact des quotas sur les inégalités régionales.

À l'intérieur des régions, les fortes inégalités entre les villes et les zones rurales persistent, les premières étant mieux dotées en infrastructures scolaires que les secondes. Si les divers plans d'éducation mis en place depuis des décennies ont, dans la plupart des régions, contribué à réduire cet écart pour l'enseignement primaire, il n'en est pas de même du secondaire qui, peu développé jusque récemment, l'était surtout dans les zones urbaines. Selon les données de l'Enquête sur le budget des ménages 2000-2001, le taux de scolarisation des citadins était sept fois plus élevé que celui des enfants des zones rurales pour les quatre années du premier cycle du secondaire, et plus de dix fois plus élevé pour les deux années du cycle supérieur du secondaire [citée par Wegdwood 2005, p. 33]. Ces différences sont également perceptibles s'agissant des taux nets de scolarisation à 
l'école secondaire entre les villes et les zones rurales, qui étaient en 2004-2005 de $18,9 \%$ en ville contre $3,1 \%$ dans les zones rurales et respectivement de $85 \%$ et $70 \%$ pour la scolarisation primaire [NBS, ORC Macro, 2004, p. 16]. En dehors de la faiblesse de ces taux, comme on le verra à travers l'exemple des Maasai vivant dans des zones rurales isolées, la qualité de l'école est également moindre : les locaux, le matériel pédagogique, le ratio enseignant-élèves ainsi que le niveau de formation des enseignants sont souvent moins bons en milieu rural. Ceci a pour conséquence un taux de réussite inférieur à l'examen de fin de cycle primaire (PLSE) des élèves ruraux, ou l'obtention de moins bonnes notes en cas de réussite. Selon Wedgwood, en 1998, le taux de réussite à l'examen PLSE était de $7 \%$ dans les zones rurales contre $29 \%$ en zone urbaine [Wedgwood, 2005, p. 13]. Ces moindres performances influent sur le type d'établissement secondaire fréquenté puisque comme nous l'avons expliqué, les places y sont essentiellement attribuées en fonction des notes obtenues à l'examen. Ainsi, les élèves scolarisés dans une école primaire rurale qui parviennent à intégrer le secteur secondaire public le font, la plupart du temps, au sein d'écoles communautaires, moins réputées et moins bien dotées que les écoles publiques régionales.

Les difficultés qui prévalent dans l'enseignement primaire sont accentuées dans les écoles secondaires communautaires, qui dépendent de la capacité de financement des habitants et n'attirent pas les enseignants les plus formés. Comme le souligne un rapport du ministère : «La pénurie générale d'enseignants est encore compliquée par le fait que les enseignants qui sont disponibles tendent à se détourner des écoles rurales » [MoEVT, 2010b, p. 33]. Ainsi, en dépit de l'existence d'un plan qui prévoit un temps de formation des futurs enseignants adapté au niveau scolaire qu'ils ont atteint et au cycle dans lequel ils sont amenés à enseigner, certaines écoles secondaires créées après 2005 - notamment celles qui se situent dans des régions reculées, difficiles d'accès et peu attractives pour les enseignants qui n'en sont pas originaires - sont dépourvues de professeurs diplômés et formés, le personnel enseignant étant constitué de personnes ayant quitté l'école après la quatrième année du secondaire sans avoir obtenu de diplôme [Lassibille, Tan, Sumra, 2000 ; Wedgwood, 2005 ; Sumra, Rajani, 2006 ; Woods, 2008]. Contrairement à ce qui avait été affiché dans le plan de 2004 (SEDP), peu de mesures incitatives ont été déployées pour encourager les enseignants à occuper des postes dans les zones reculées du pays. Comme le souligne Woods, «Les stratégies utilisées pour pourvoir des postes en milieu rural ont été d'y envoyer des stagiaires en première année de formation à l'institut de formation des enseignants en espérant qu'ils resteraient. Il en résulte une mauvaise prise en charge des stagiaires, de nombreux abandons et une mauvaise qualité de l'éducation » [2008, p. 428].

La réduction des inégalités entre les régions et entre les zones rurales et urbaines repose donc essentiellement sur le développement - quantitatif - de l'offre scolaire et plus précisément, sur la construction d'écoles secondaires communautaires. Or la décision de bâtir une école communautaire est prise au sein des comités de quartiers, qui se chargent ensuite de collecter des fonds pour 
l'achat des matériaux et de rassembler la main-d'œuvre pour bâtir l'édifice. Les subventions du gouvernement qui servent à viabiliser l'école parviennent en bout de chaîne et leurs montants, voire l'effectivité du paiement lui-même, varient d'un endroit à l'autre. Mais plus encore que ces variations d'allocations, l'aptitude des communautés à collecter des fonds pour la construction d'une école est inégalement répartie sur l'ensemble du territoire. Ainsi, la présence même d'un établissement secondaire et les conditions de scolarisation qu'il sera susceptible d'offrir dépendent-elles essentiellement de la volonté et de la capacité de financement des districts et, en leur sein, des cantons. Les régions les plus reculées ou les plus pauvres, celles qui sont précisément ciblées par une telle mesure, sont aussi celles qui ont le plus de difficultés à en financer la construction et surtout à les entretenir ensuite.

\section{Évolution et conditions de scolarisation des Maasai}

\section{L'augmentation de l'offre scolaire}

Les Maasai figurent parmi les populations les moins scolarisées du pays. Pasteurs semi-nomades vivant en habitat dispersé dans des zones difficiles d'accès et pauvres en infrastructures scolaires, ils ont eux-mêmes été pendant longtemps peu enclins à scolariser leurs enfants, auxquels ils souhaitaient transmettre une éducation pastorale. Cette dernière, qui s'acquiert surtout par l'expérience du gardiennage des troupeaux, se révèle à la fois utile à l'économie de la famille - la division du travail pastoral reposant sur la main-d'œuvre enfantine pour mener les troupeaux en pâture - et nécessaire à leur future vie d'adulte maasai. Spécialisée et de longue haleine, l'éducation pastorale occupe les enfants pendant les mêmes heures de la journée que l'école [Bonini, 1996]. À la fin des années 1970, la volonté d'amener les pasteurs à délaisser leurs modes de vie et de production, jugés obsolètes et peu productifs, et de parvenir à l'éducation universelle a amené le gouvernement tanzanien à développer l'offre scolaire dans les zones faiblement scolarisées. De nombreuses écoles construites dans la région à cette période ont alors été fréquentées par les enfants de pasteurs. Les enquêtes que nous avons menées en 1991 et 1993 dans les districts de Monduli et de Ngorongoro faisaient apparaître un taux de scolarisation primaire des enfants maasai d'environ $33 \%$ contre une moyenne nationale aux alentours de $68 \%$ à cette époque - tandis qu'une infime partie d'entre eux continuait leurs études au-delà du primaire [Bonini, 1996]. Certains quittaient l'école avant la dernière année, mais la plupart d'entre eux poursuivaient leur scolarité jusqu'à l'examen de fin de cycle - qu'ils n'obtenaient pratiquement jamais - et menaient ensuite une vie similaire à celle de leurs camarades non scolarisés; les filles étaient rapidement mariées, tandis que les garçons débutaient leur vie de guerriers ${ }^{13}$. Dans la mesure où dans leur immense majorité, les enfants restaient dans leur village après avoir terminé leur

13. Ce terme (traduction du terme moran en maa) désigne la première classe d'âge qui regroupe les jeunes hommes initiés. 
scolarité primaire, les parents préféraient envoyer leurs garçons à l'école, pour bénéficier des fruits de leur éducation ${ }^{14}$. Jusqu'au début des années 1990, la scolarisation des enfants n'avait que peu d'impact sur les conditions et le mode de vie des Maasai. Elle n'engendrait pas, ou de façon très marginale, d'exode rural des jeunes. L'école était davantage jugée sur sa capacité d'alphabétisation en swahili que sur les débouchés offerts. En raison de la faiblesse du taux de réussite à l'examen de fin de primaire et de celle des infrastructures scolaires secondaires dans la région d'une part, et de la volonté des familles de profiter de l'instruction en swahili de leurs enfants d'autre part, la mobilité sociale et géographique des enfants était à la fois faible et non souhaitée.

En 2009, lors de notre second séjour dans les districts où avaient été réalisées les enquêtes de 1993, l'offre scolaire dans la région avait sensiblement évolué : alors qu'en 1993, on comptait 74 écoles primaires dans les deux districts (47 à Monduli et 30 à Ngorongoro), leur nombre avait atteint 141 (dont 6 écoles privées) en 2008 (33 dans le district de Longido ${ }^{15}$, 49 dans celui de Monduli et 59 dans celui de Ngorongoro). L'augmentation des effectifs scolaires primaires et secondaires (décrits précédemment à l'échelle du pays) est également perceptible, bien que de plus faible ampleur sur le territoire rural des Maasai. L'origine ethnique des élèves n'étant pas mentionnée dans les documents officiels, il est difficile de déterminer avec exactitude le pourcentage d'enfants maasai scolarisés. Selon les statistiques régionales, le taux net de scolarisation primaire dans les districts de Monduli et Ngorongoro, était respectivement de 84,9\% et de 83,7\% en 2008 [MoEVT, 2010] ${ }^{16}$. De même, bien qu'ils ne fournissent qu'une indication, les entretiens que nous avons effectués en 2009 avec des parents d'élèves et des enseignants, ainsi qu'une enquête réalisée en 2004-2005 dans le nord du district de Monduli par E. Bishop, qui estime à $50 \%$ la population maasai scolarisée à l'école primaire [Bishop, 2006, p. 4], vont également dans le sens d'un accroissement du nombre d'élèves maasai.

S'agissant de l'enseignement secondaire, en 1993, une seule école secondaire publique - ouverte cette même année - et une école privée étaient présentes dans les deux districts ; en 2008, on comptait 30 établissements secondaires (dont 24 publics). Le nombre d'écoles secondaires s'est accru depuis 2007 avec l'implantation d'écoles communautaires, dont la très grande majorité s'arrête après la quatrième année. À titre d'exemple, sur les 18 écoles secondaires du district de Monduli, dont cinq sont privées, seules trois écoles - dont une seule gouvernementale - proposent un enseignement sur les six années du secondaire. Lors des

14. En effet, en vertu du mode de résidence patrilocal, les filles suivent leur époux. Seuls quelques parents réticents à la scolarisation qui préféraient envoyer les filles à l'école pour répondre à l'obligation scolaire tout en gardant leurs fils comme bergers.

15. Le district de Longido est né de la scission du district de Monduli au début des années 2000.

16. En dehors des écoles situées dans les petites villes des chefs-lieux de district, la majorité des écoles rurales de ces districts est fréquentée par des enfants maasai. La proportion se réduit au secondaire ; une partie des établissements secondaires possédant des internats, ils accueillent également des enfants d'autres districts, voire d'autres régions, qui peuvent être ou ne pas être maasai. 
enquêtes de terrain de 2009, huit établissements n'avaient pas encore d'élèves dans le niveau 4 puisqu'ils avaient ouvert en 2007, d'abord avec les élèves de la première année uniquement, parvenus en deuxième année l'année suivante, et, pour ceux qui avaient réussi l'examen de fin de deuxième année, en troisième année en $2009^{17}$. Toujours moins représentés que leurs concitoyens sur les bancs du secondaire, les Maasai sont désormais effectivement présents à ce niveau : ils constituaient en 2009 environ la moitié des 400 élèves des deux écoles secondaires d'Engaruka et de Selela, deux villages du district de Monduli.

\section{La permanence des mauvaises conditions de scolarisation}

$\mathrm{Si}$, en termes d'infrastructures scolaires le territoire maasai est aujourd'hui bien mieux couvert qu'il ne l'était il y a une décennie, les conditions de scolarisation et la qualité de l'éducation dispensée ne se sont guère améliorées. Les études et rapports sur l'éducation en Tanzanie ${ }^{18}$ comme nos récentes enquêtes de terrain révèlent la permanence des mauvaises conditions de scolarisation tant au niveau primaire que dans les écoles secondaires. Dans la très grande majorité des écoles visitées - dans les années 1990 comme en 2009 - les locaux (salles de cours, internats, logements des instituteurs) sont insuffisants et en mauvais état, et le matériel scolaire fait défaut : il se limite au cahier et au stylo de l'enfant ou, au mieux et, pour certaines leçons uniquement, à un livre à partager. La récitation à l'oral des leçons que l'enseignant (ou un élève) inscrit au tableau constitue la méthode principale d'apprentissage. En dehors de ces carences matérielles et pédagogiques, l'insuffisance du nombre d'enseignants dans les régions rurales isolées et/ou leur fort absentéisme font que les élèves passent de nombreuses heures seuls dans les classes. Les motifs d'absence des enseignants sont variés : en dehors de la maladie et des raisons personnelles, ils pratiquent souvent une autre activité pour subvenir à leurs besoins, ce qui peut les éloigner provisoirement de leur salle de cours. Ils sont également régulièrement sollicités par leur autorité de tutelle à se rendre dans le chef-lieu du district, pour des raisons administratives liées à leur situation personnelle ou à celle de l'école; l'éloignement et les difficultés de transport augmentent le temps passé loin de la classe. Les conditions de vie et de travail des enseignants comme de leurs élèves sont difficiles : construits rapidement, les bâtiments ne sont souvent pas terminés, les logements pour les enseignants sont insuffisants, de même que les dortoirs pour les élèves. Comme le déplorait l'un des enseignants d'une école secondaire communautaire construite en 2007 : «Avec la venue de nouveaux élèves l'un prochain, nous serons certainement obligés de transformer l'une des salles de classe en dortoir pour filles la

17. D'après la responsable du service régional de l'éducation d'Arusha interrogée en août 2009, cette même année avait connu un afflux important d'élèves en première année du secondaire correspondant à celui qu'avait connu la première année du primaire sept ans plus tôt, lors de l'abolition des droits d'inscription; les élèves étaient ainsi beaucoup plus nombreux à avoir passé l'examen de fin de primaire et mécaniquement plus nombreux à l'avoir réussi.

18. Cf. notamment Davidson [2004], HakiElimu [2009], Lassibille, Tan, Sumra [2000], Sumra, Rajani [2006], Wedgwood [2005], et Woods [2008] pour la Tanzanie ; Bugeke [1997], Carr-Hill, Peart [2005], et Mwegio, Mlekwa [2002] pour le pays maasai. 
nuit et de ranger les lits pour installer les tables le jour. » (enseignant de l'école secondaire d'Engaruka, Engaruka, 2009. Deux des écoles secondaires visitées en août 2009 subissaient une pénurie d'eau depuis plusieurs semaines. Dans l'une d'elles, élèves et enseignants avaient passé de nombreuses journées à aller chercher de l'eau avant de décider de creuser une tranchée pour y faire passer un tuyau, activité qui les avait occupés plus de 15 jours, au détriment de leurs cours. Ces situations ne sont pas rares et provoquent des abandons dans les rangs des élèves, parfois des rancœurs parmi les habitants et les parents qui ont financé le bâtiment et des demandes de mutation de professeurs. Étant donné le très faible nombre de Maasai ayant achevé l'école secondaire jusqu'à ces dernières années, ils sont rares à pouvoir enseigner, à fortiori à ce niveau. Or comme le signalait l'un des enseignants rencontrés, « seuls les Maasai aiment travailler ici » (enseignant de l'école secondaire d'Engaruka, Engaruka, 2009).

Les mauvaises conditions de scolarisation ne sont pas propres au pays maasai. Elles sont ici, comme dans d'autres zones reculées, accrues par un environnement géographique hostile, cette région semi-aride connaissant de nombreuses sécheresses et un environnement social et économique peu favorable. La paupérisation de nombreux Maasai, essentiellement due à la détérioration des conditions d'exercice du pastoralisme qui s'est amplifiée depuis plus d'une décennie ${ }^{19}$, et l'hostilité ou, le plus souvent, l'indifférence à l'égard de l'école de certains, ne favorisent pas l'investissement dans la construction ou la rénovation d'écoles. En définitive, si les enfants Maasai sont plus nombreux à être scolarisés qu'auparavant, ils continuent de l'être dans des conditions médiocres. Celles-ci ont une incidence à la fois sur la qualité de l'apprentissage, sur la maîtrise des connaissances acquises et sur les performances scolaires des élèves. Leurs chances d'accéder à l'enseignement secondaire public, d'une part, et d'être inscrits dans des établissements mieux dotés et plus réputés d'autre part, sont amoindries, ce qui diminue de fait les possibilités d'accéder à l'enseignement supérieur. Toutefois, les conditions de scolarisation ne sont pas seules en cause : l'incapacité des familles à financer la scolarité de leurs enfants, notamment au-delà du primaire, représente également un frein important.

\section{Scolariser pour diversifier les ressources}

Le développement de l'offre scolaire, les incitations à la scolarisation de divers acteurs, (églises, instituteurs, élus locaux, ONG, Maasai éduqués), le don d'un repas par le World Food Programme dans de nombreuses écoles reculées, et surtout le déclin du pastoralisme engendrant à la fois la nécessité de diversifier les sources de revenus, la perte de confiance dans la viabilité de ce mode de production, et la vacance des gardiens de troupeaux, ont conduit les Maasai à davantage scolariser leurs enfants. Dans les années 1990, l'école était globalement considérée

19. Sur la détérioration du pastoralisme en général et chez les Maasai en particulier, $c f$. Anderson, Broch-Due [1999], Dyer [2006], Fratkin, McCabe [1999], Kituyi [1990], Krätli [2000], Ndagala [1995], Åhrem [1985]. 
comme un moyen d'intégration dans la société globale, et les parents comptaient sur le retour de leurs enfants pour pouvoir profiter de leurs connaissances en swahili. Comme le montrent les entretiens que nous avons réalisés en 2009, les attentes des parents par rapport à l'école ont changé. Si la maîtrise du swahili est toujours aussi nécessaire, car elle permet les contacts avec les non-Maasai (sur les marchés, dans les hôpitaux, les administrations, les transports, etc.), elle n'est plus considérée comme suffisante, et ceux qui voyaient en elle la finalité de l'école ne sont plus nombreux. Qu'ils possèdent de vastes troupeaux ou qu'ils n'aient plus que quelques têtes de bétail, ils sont désormais plus nombreux à espérer que les enfants qu'ils scolarisent - notamment à l'école secondaire, auparavant inaccessible - trouvent un emploi en remplacement ou en complément des activités pastorales. Cette nécessité de diversifier les sources de revenu se révèle plus impérieuse pour les Maasai qui ont perdu leurs troupeaux et pour beaucoup, l'espoir de vivre du pastoralisme, d'autant qu'elle a libéré leurs enfants de l'activité de berger et rendu la transmission du savoir pastoral moins essentielle. De la même façon que les Maasai s'engagent davantage dans la scolarisation, ils sont également plus nombreux à s'impliquer dans des activités non pastorales, telles que l'agriculture ou le commerce, considérées comme des voies privilégiées pour sortir de la pauvreté et présentées, notamment via l'école, comme des facteurs de progrès. Comme le souligne un père de famille :

«Moi, je regrette aujourd'hui de ne pas avoir envoyé tous mes enfants à l'école : maintenant, c'est la sécheresse, le bétail meurt et on ne peut rien faire. On doit attendre les rations alimentaires du gouvernement. On ne peut pas avoir de travail lorsqu'on n'est pas allé à l'école. Mes derniers enfants sont à l'école et s'ils réussissent l'examen de fin de primaire, ils iront au secondaire. S'ils trouvent ensuite un travail, ils pourront nous aider. Je ferai mon possible pour qu'ils aillent au secondaire mais ce n'est pas facile car, même dans les écoles du gouvernement, il faut payer la nourriture, les uniformes, les cahiers et toutes sortes de choses. » (Père de huit enfants dont les trois derniers sont scolarisés, Engaruka, 2009).

Ainsi, la paupérisation des Maasai, qui encourage la scolarisation des enfants à l'école primaire devient ensuite un frein à la poursuite de leurs études. Autrement dit, les individus qui perçoivent la scolarisation de leurs enfants comme une alternative au mode de vie pastoral sont souvent ceux qui n'ont pas les moyens de payer les frais qu'engendre la scolarisation secondaire, surtout lorsqu'elle implique un hébergement en internat.

\section{Conclusion}

Avec un taux de scolarisation primaire proche de la scolarisation universelle et un taux de scolarisation secondaire en forte croissance, la Tanzanie figure parmi les pays qui ont le plus œuvré pour atteindre les objectifs de l'EPT et du Millénaire (OMD). Le gouvernement a centré ses efforts sur l'accès à l'école, en termes d'inscriptions, et sur la construction d'établissements scolaires, autrement dit, sur le développement quantitatif de l'éducation scolaire. L'afflux d'élèves qui en a résulté a pris de court les enseignants qui ne sont ni assez nombreux ni 
suffisamment formés pour les encadrer, notamment au niveau du secondaire. En effet, si comparativement à d'autres pays le taux de scolarisation à ce niveau est relativement bas, sa progression à partir des années 2000 a été aussi rapide qu'importante, ce qui a mécaniquement provoqué un déficit d'enseignants; ce taux étant demeuré très faible pendant de nombreuses années, peu d'individus ont le niveau requis pour enseigner dans le secondaire. Cette progression est essentiellement due à la création d'écoles secondaires communautaires, particulièrement dans les districts et cantons où elles faisaient défaut, ce qui a contribué à réduire le fossé entre les régions favorisées et les autres, comme entre les villes et les campagnes. Il reste que nombre de ces établissements, notamment ceux situés dans les zones rurales isolées, n’ont pas les moyens humains ni matériels de dispenser une éducation de qualité qui puisse favoriser la réussite des élèves aux examens de fin de cycle, ni même, selon certains auteurs, l'acquisition de connaissances utiles pour leur vie future [HakiElimu, 2009; Sumra, Rajani, 2006 ; Woods 2008]. Du côté des familles, l'implantation des écoles secondaires dans un grand nombre de cantons a réduit les coûts de transport et d'hébergement que pouvait engendrer la scolarisation post-primaire. En outre, le fait que cette expansion soit essentiellement due à la création d'écoles publiques a limité les frais de scolarité, supérieurs dans le secteur privé. Elle a en revanche fait reposer sur les habitants, donc collectivement sur les familles, les coûts de construction des bâtiments, et sur les parents d'élèves, ceux du matériel scolaire et pédagogique comme du fonctionnement de l'école et, le cas échéant, de l'internat. Ces récentes écoles accueillent une partie des enfants maasai qui sont par ailleurs plus nombreux que dans les années 1990 à obtenir l'examen de fin de cycle primaire. Cependant, tandis que grâce notamment à la possibilité accrue de poursuite d'étude et aux débouchés que l'école est susceptible d'offrir, leurs parents envisagent davantage de les scolariser au-delà du primaire - ou du moins une partie d'entre eux - la faible qualité de l'enseignement dispensé et le coût global de la scolarisation, difficilement supportable pour certains ménages, augmentent les risques d'échec et d'abandons des élèves.

\section{Bibliographie}

ÅHREM K. [1985], "Pastoral Maasai in the garden of Eden. The Maasai of the Ngorongoro Conservation Aera, Tanzania", Research Reports in Cultural Anthropology, vol. 3, Uppsala, University of Uppsala, $123 \mathrm{p}$.

Al-Samarrai S., Reilly B. [2005], "Education, Employment and Earnings of Secondary School-Leavers in Tanzania: Evidence from a Tracer Study", PRUS Working paper, $\mathrm{n}^{\circ} 31$, 42 p. Brighton, University of Sussex : http://www.sussex.ac.uk/Units/PRU/wps/wp31.pdf (page consultée le 19 janvier 2011).

Anderson D.M., Broch-Due V. [1999], The Poor Are Not Us: Poverty and pastoralism, Oxford, James Currey, $356 \mathrm{p}$. 
Bishop E. [2006], "The Policy and Practice of Educational Service for Pastoralists in Tanzania”, Research Conference on Pastoralism and Poverty Reduction in East Africa, Organized by the International Livestock Research Institute (ILRI), June 27-28, 2006, Nairobi, Kenya, 15 p. : http://www.saga.cornell.edu/saga/ilri0606/22bishop.pdf (page consultée le 12 février 2011).

BoninI N. [1996], «Éducation non scolaire et école primaire : les conséquences d'une rencontre. Une étude anthropologique de la transmission du savoir chez les Maasai de Tanzanie », Doctorat d'anthropologie sociale, Paris, École des hautes études en sciences sociales, 466 p.

Bonini N. [2003], «Un siècle d'éducation scolaire en Tanzanie », in LANGE M.-F., « Enseignements », Cahiers d'études africaines, 2003/1-2, n 169-170, p. 41-62.

BugeKe C.J. [1997], « Nomadic Education in Tanzania », in Ezeomah C. (ed.), The Education of Nomadic Populations in Africa, Papers presented at the Unesco (Breda) regional seminar on the education of nomadic people in Africa, 11-15 December 1995, Kaduna, Nigeria, Dakar, BREDA-Unesco.

Carr-Hill R., Peart E. [2005], The education of nomadic peoples in east Africa: Djibouti, Eritrea, Ethiopia, Kenya, Tanzania and Uganda, Review of Relevant Literature, Paris, Unesco, International Institute for Educational Planning, $138 \mathrm{p}$.

Charton H. [2003], « La débâcle éducative au Kenya », in Lange M.-F., «Enseignements », Cahiers d'études africaines, 2003/1-2, n ${ }^{\circ}$ 169-170, p. 189-208.

DAVIDSON E. [2004], "The progress of the primary education development plan (PEDP) in Tanzania: 2002-2004”, HakiElimu Working Paper Series, 2004/2, Dar Es Salaam, HakiElimu, $14 \mathrm{p}$.

DYer C. [2006], The Education of Nomadic Peoples: Current Issues, Future Prospects, Oxford, New York, Berghahn Books, 278 p.

Forum MONDIAL SUR L'ÉDUCATION [2000], Cadre d'action de Dakar-L'Éducation pour tous : tenir nos engagements collectifs, Texte adopté par le Forum mondial sur l'éducation, Dakar, Sénégal, 26-28 avril 2000, Paris, Unesco, 78 p. : http://unesdoc.unesco.org/images/ 0012/001211/121147f.pdf (page consultée le 5 février 2011).

Fratkin E. M., McCabe J.T. (eds.) [1999], "East African Pastoralists at the crossroads", Nomadic People, Special Edition, vol. 3, n 2, 182 p.

HaKielimu [2009], Annual Report 2009, Dar es Salaam, HakiElimu, 48 p. : http://www.hakielimu.org (page consultée le 11 avril 2011).

KituYi M. [1990], Becoming Kenyans. Socio-economic Transformation of the Pastoral Maasai, Nairobi, Act Press, African centre for Technology studies, 251 p.

KRÄTLI S. [2000], "Education provision to nomadic pastoralists: A literature review”, IDS Working paper, $\mathrm{n}^{\circ}$ 126, Brighton, World Bank-IDS, University of Sussex, UK, 84 p.

Lassibille G., TAN J.-P., SumRa S. [2000] "Expansion of Private Secondary Education: Lessons from Recent Experience in Tanzania", Comparative Education Review, vol. 44, n ${ }^{\circ}$ 1, p. 1-28.

Ministry of EDUCATION AND VOCATIONAL TRAINING (MoEVT) [2010a], Basic Education Statistics in Tanzania : http://educationstatistics.moe.go.tz/moe/(page consultée le 11 avril 2011).

Mwegio L., Mlekwa V. M. [2002], Education for nomadic communities in Tanzania. Dar Es Salaam, Unicef/Unesco, Ministry of Education and Culture.

Ndagala D. K. [1995], "Pastoral Territory and Policy Debates in Tanzania", Nomadic Peoples, $\mathrm{n}^{\mathrm{o}}$ 34-35, p. 23-36.

SAmoff J. [1987], "School Expansion in Tanzania: Private Initiatives and Public Policy", Comparative Education Review, vol. 31, nº 3, p. 333-360. 
Sumra S., Rajani R. [2006], "Secondary Education in Tanzania: Key Policy Challenges", HakiElimu Working Paper, 2006/4, Dar Es Salaam, HakiElimu, 8 p.

Ministry OF EDUCATION AND CULTURE (MEC) [2000], Secondary Education Master Plan (SEMP) 2001-2005, Dar es Salaam, 53 p.: http://moe.go.tz/pdf/SEMP\%202001\%202005\%20ver II.pdf (page consultée le 4 avril 2011).

MinistRy OF EDUCATION AND CULTURE (MEC) [2001], Teacher Education Master Plan (TEMP) 2000-2005, Dar es Salaam, 94 p. : http://moe.go.tz/pdf/TEMP.pdf (page consultée le 4 avril 2011).

Ministry of EDUCATION AND CULTURE (MEC) [2004], Secondary Education Development Plan (SEDP) 2004-2009, Dar es Salaam, 60 p. : http://moe.go.tz/pdf/SEDP.pdf (page consultée le 12 mars 2011).

Ministry of education and vocational training (MoEVT) [2010b], Secondary Education Development Programme II (July 2010-June 2015), Dar es Salaam, 94 p. : http://moe.go.tz/ PDF/SEDP2010.pdf (page consultée le 11 mars 2011).

National bureau of STAtistics (NBS), ORC Macro [2005], Tanzania Demographic and Health Survey (DHS) (2004-2005), Dar es Salaam, Tanzania, National Bureau of Statistics and ORC Macro, 381 p. : http://rochr.qrc.com/bitstream/123456789/311/1/2004\%20Tanzania\% 20DHS\%20report.pdf (page consultée le 14 février 2011).

Wedgwood R. [2005], "Post-Basic Education and Poverty in Tanzania", Post-Basic Education and Training Working Paper Series, $\mathrm{n}^{\circ}$, Centre of African Studies, University of Edinburgh, $56 \mathrm{p}$.

Woods E. [2007], “Tanzania country case study", Country profile prepared for the Education for All Global Monitoring Report 2008. Education for All by 2015 : will we make it? 2008/ED/ EFA/MRT/PI/79, 36 p. : http://unesdoc.unesco.org/images/0015/001555/155592e.pdf.

Woods E. [2008], "Progress towards EFA in Tanzania", Prospects, vol. 38, no 3, "Education for All by 2015: Progress and Challenges", p. 425-430. 